\title{
STRUCTURAL CHARACTERIZATION OF LIGHT METAL BOROHYDRIDES BY DISPERSION-CORRECTED DENSITY FUNCTIONAL THEORY MODELLING
}

\section{Mariana Derzsi, ${ }^{1,3}$ A. Starobrat, ${ }^{2,3}$ T. Jaroń, ${ }^{3}$ P. Malinowski ${ }^{3}$ and W. Grochala ${ }^{3}$}

${ }^{1}$ ATRI Institute Materal-Tech. Faculty, Slovak University of Technology in Bratislava

${ }^{2}$ College of Inter-Faculty Individual Studies in Math. and Natural Sciences, University of Warsaw

${ }^{3}$ Center of New Technologies, University of Warsaw

mariana.derzsi@stuba.sk
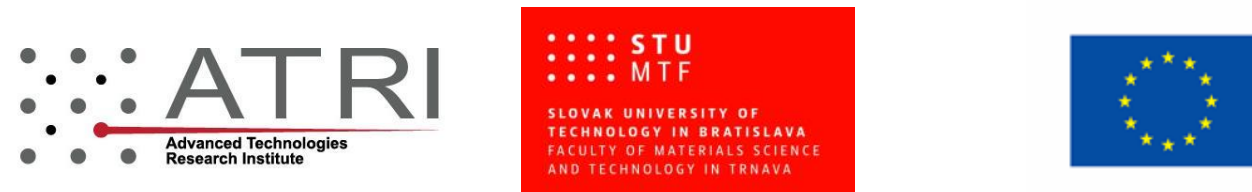


\section{METAL BOROHYDRIDES}

\section{Importance}

- H-rich systems with: potential hydrogen storage material

\section{Challenges}

- Thermodynamic stability:

- $\mathrm{Be}\left(\mathrm{BH}_{4}\right)_{2}$ with 20.7 wt.\% $\leftarrow$ immensely toxic

- $\mathrm{Al}\left(\mathrm{BH}_{4}\right)_{3} 18.4$ wt.\% $\%$ explosive

- $\mathrm{Mg}\left(\mathrm{BH}_{4}\right)_{2} 14.8 \mathrm{wt} . \% \quad \leftarrow$ overly-stable

\section{Solutions}

- Synthesis of multi-cation borohydrides.

- Structural systematics challenging: low crystallinity, poorly scattering atoms, frequent substitutional disorder.

- Computational modelling as a great aid to structure characterization. 


\section{Mixed metal borohydride $\mathrm{LiSc}\left(\mathrm{BH}_{4}\right)_{4}$}

- The first tetragonal $\alpha$ polymorph $(P-42 \mathrm{c}, \mathrm{Z}=2)$ was reported in 2008.

- In 2018, tetragonal $\boldsymbol{\beta}$ phase was observed $(/ 4 / \mathrm{m}, \mathrm{Z}=8)$.

- Recently, cubic $\boldsymbol{y}$ polymorph $(P-43 \mathrm{~m}, \mathrm{Z}=1)$ proposed.

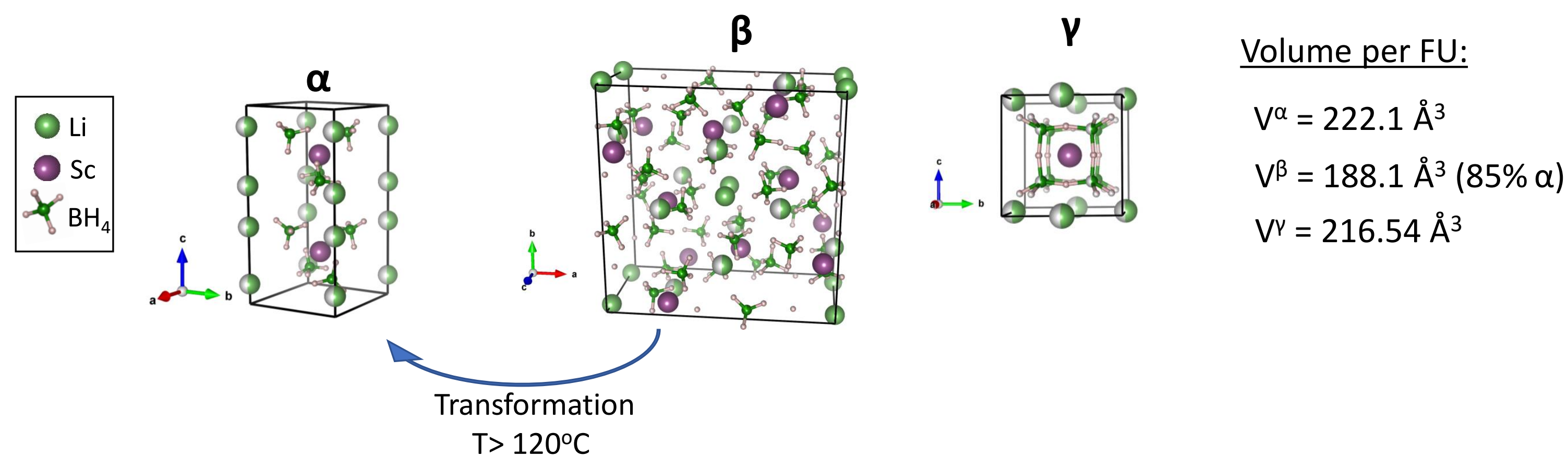




\section{$\alpha-\operatorname{LiSc}\left(\mathrm{BH}_{4}\right)_{4}$}

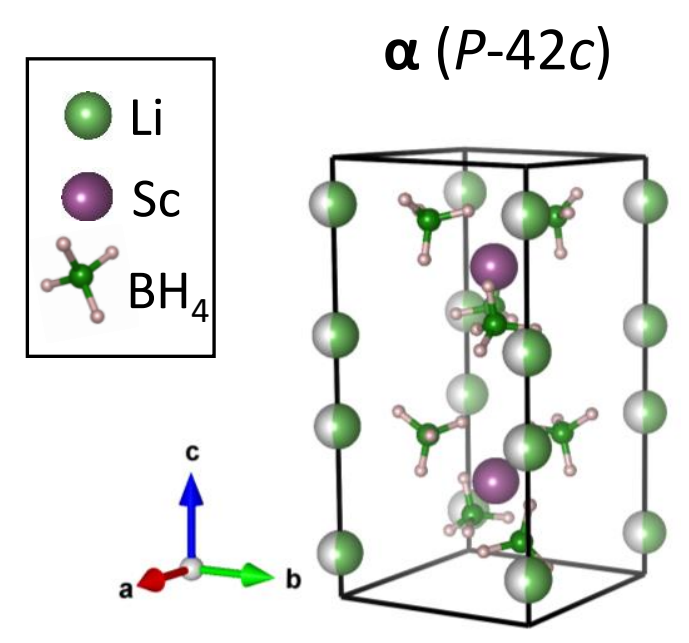

Synchrotron measurement

Hagemann et al., J. Phys. Chem. A (2008) ordered $\boldsymbol{\alpha}$ model (P222 $)$

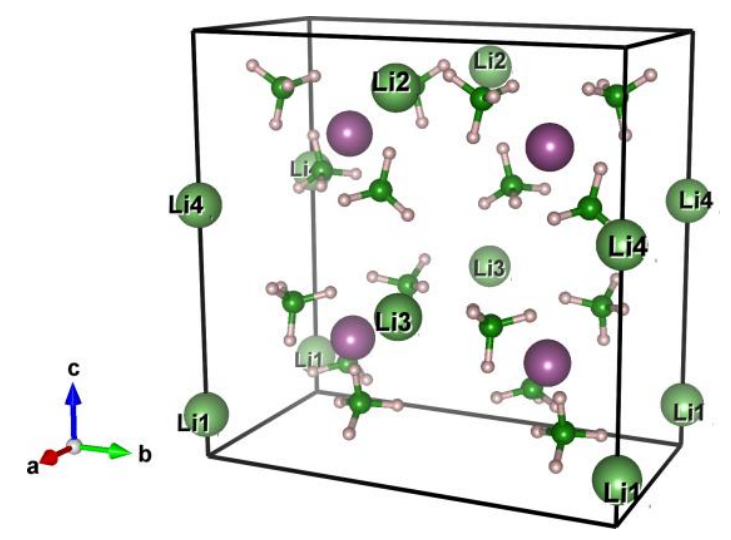

Predicted ground state (I-4)

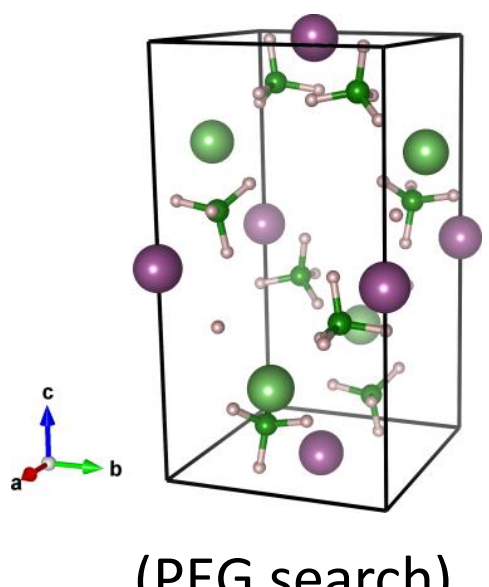

(PEG search)
- Kim's models do not match the diffraction pattern of $\boldsymbol{\alpha}$

Kim et al., J. Phys. Chem. C (2009) 


\section{$\beta-\operatorname{LiSc}\left(\mathrm{BH}_{4}\right)_{4}$}
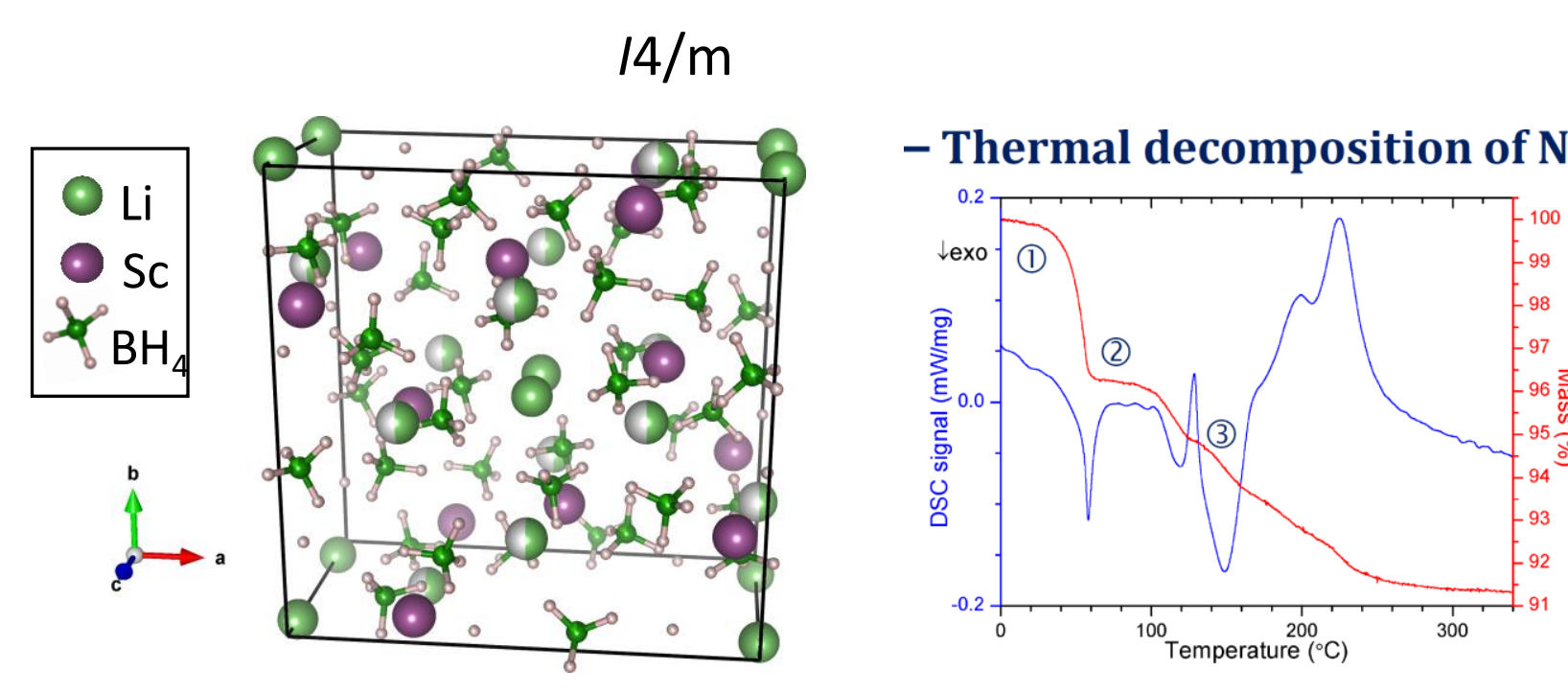

Crystalline products:

(1) $-\mathrm{NH}_{4} \mathrm{Sc}\left(\mathrm{BH}_{4}\right)_{4}$ and $\mathrm{LiCl}$

(2) - $\boldsymbol{\beta}-\mathbf{L i S c}\left(\mathrm{BH}_{4}\right)_{4}$ and $\mathrm{LiCl}$

new polymorph!

(3) $-\boldsymbol{\alpha}-\mathbf{L i S c}\left(\mathrm{BH}_{4}\right)_{4}$ and $\mathrm{LiCl}$

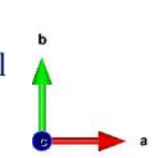

Structural similarities with $\mathrm{NH}_{4} \mathrm{Sc}\left(\mathrm{BH}_{4}\right)_{4}$ precursor

Starobrat, T. Jaroń, W. Grochala, Dalton Trans. (2018)

$$
\begin{array}{cl}
\boldsymbol{\beta}-\operatorname{LiSc}\left(\mathrm{BH}_{4}\right)_{4} & \mathrm{NH}_{4} \mathrm{Sc}\left(\mathrm{BH}_{4}\right)_{4} \\
\mathrm{a}=14.3 \AA & \mathrm{a}=15.7 \AA \\
\mathrm{c}=7.4 \AA & \mathrm{c}=7.9 \AA \\
\beta=90.5^{\circ} & \alpha=89.5^{\circ} \\
& \beta=90.5^{\circ} \\
& \gamma=81.4^{\circ} \\
& \text { (in } \beta \text {-LiSc }\left(\mathrm{BH}_{4}\right)_{4} \\
& \text { representation) }
\end{array}
$$




\section{$\beta-\operatorname{LiSc}\left(\mathrm{BH}_{4}\right)_{4}-$ DFT results}

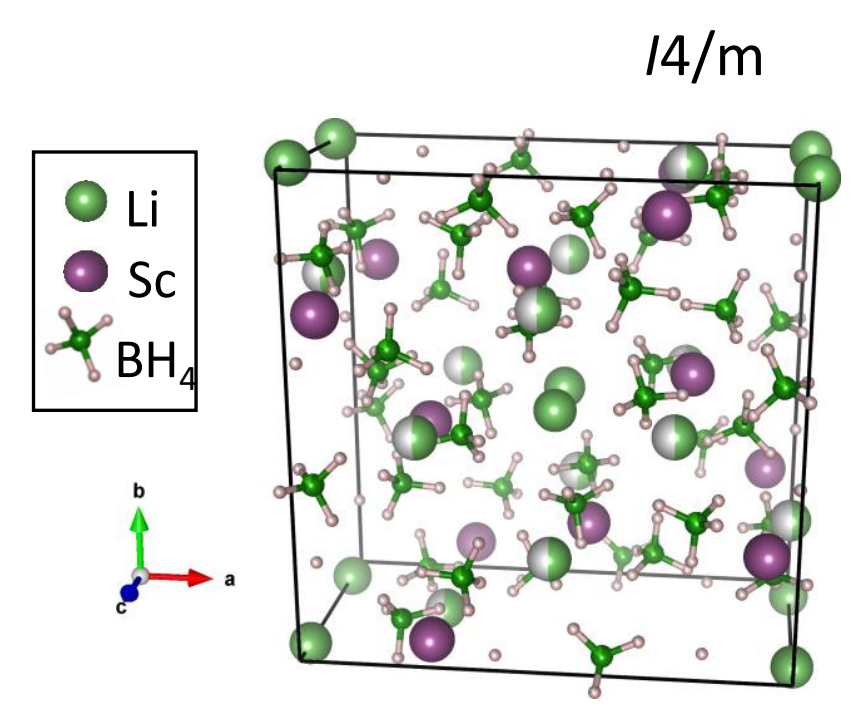

1. Ordered $\beta$ models
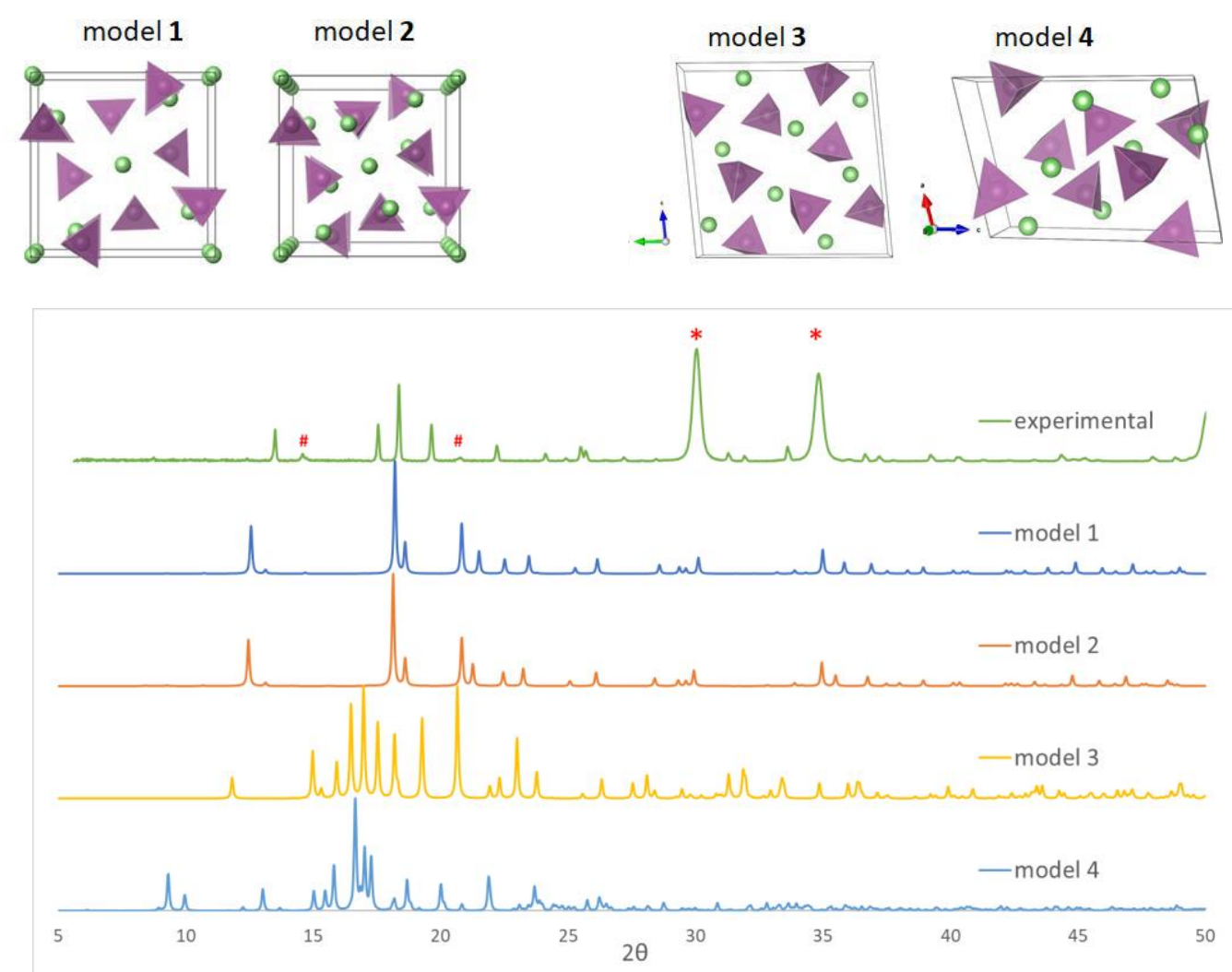

2. $\mathrm{NH}_{4} \mathrm{Sc}\left(\mathrm{BH}_{4}\right)_{4}$ type models

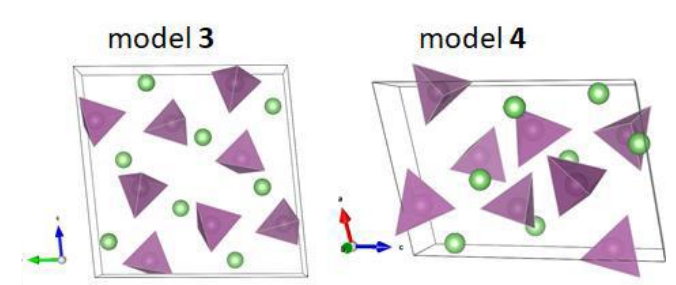

- Many ordered models were tested.

- Our approach to describe $\beta$ phase with ordered models has failed. 


\section{Common features of $\alpha$ and $\gamma \operatorname{LiSc}\left(\mathrm{BH}_{4}\right)_{4}$}
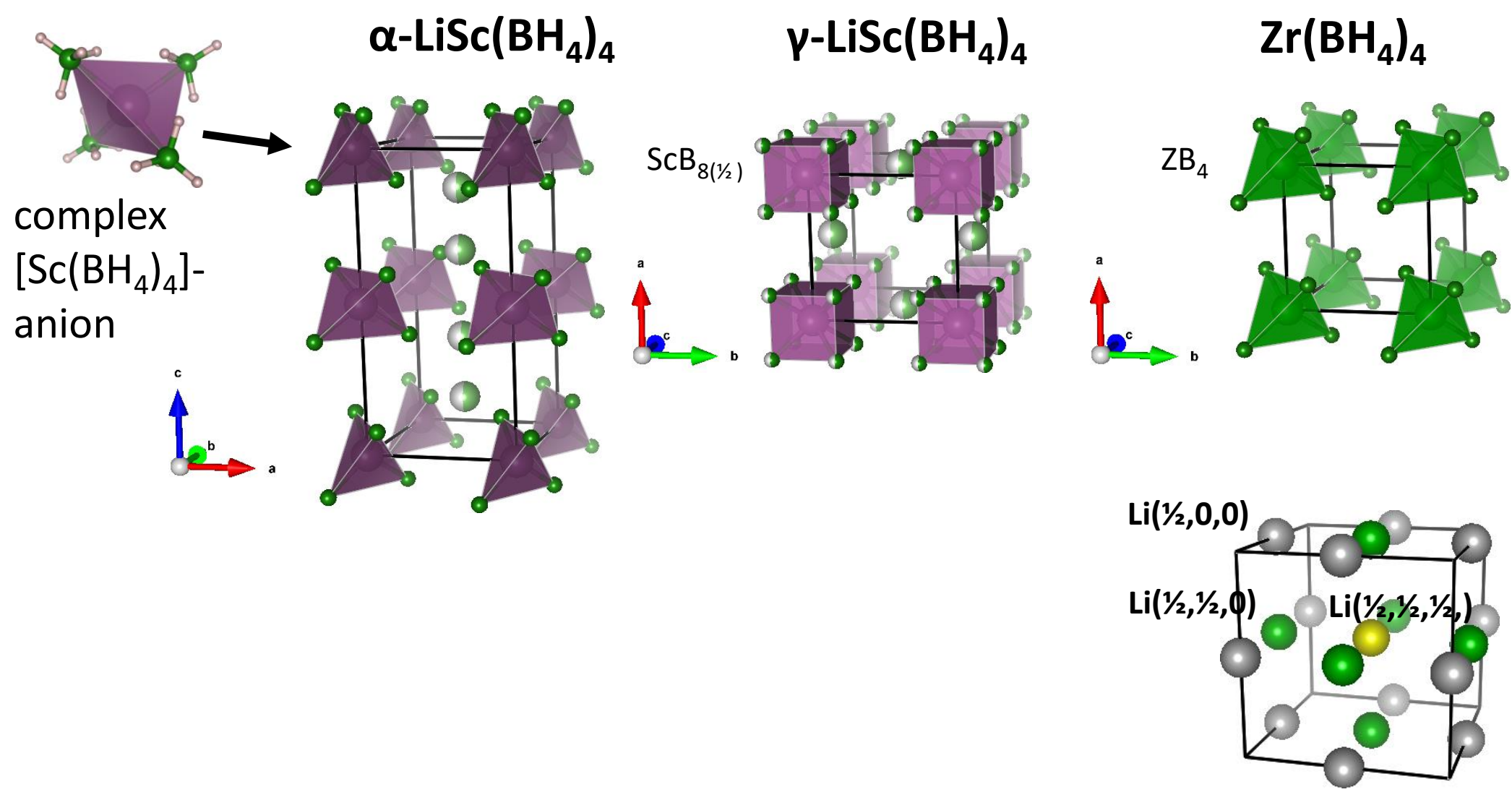

- $\alpha$ and $\boldsymbol{\gamma}$ phase share simple cubic Sc sublattice

- Differ only in occupation of interstitial positions by Li

- $\mathrm{Zr}\left(\mathrm{BH}_{4}\right)_{4}$ prototype structure contains tetrahedral voids ready to host Li in:

- Centre $(1 / 2,1 / 2,1 / 2)$

- Face $(1 / 2,1 / 2,0)$

- Edge $(1 / 2,1 / 2,1 / 2)$ 


\section{DFT Modelling $\mathrm{LiSc}\left(\mathrm{BH}_{4}\right)_{4}$ in $\mathrm{Zr}\left(\mathrm{BH}_{4}\right)_{4}$ type lattice}

\section{Modelling strategy}
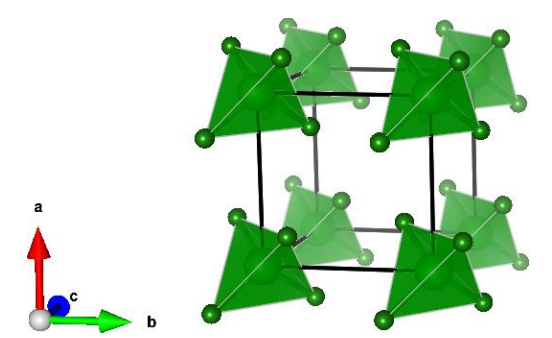

$\underset{\mathrm{Li} \rightarrow \text { tetrahedral vacancy }}{\longrightarrow}$

$$
\mathrm{Zr}\left(\mathrm{BH}_{4}\right)_{4}
$$
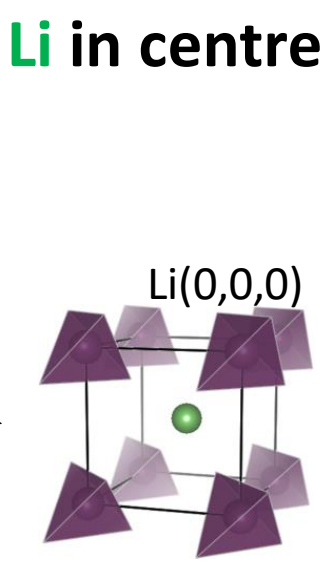

DFT-D3:

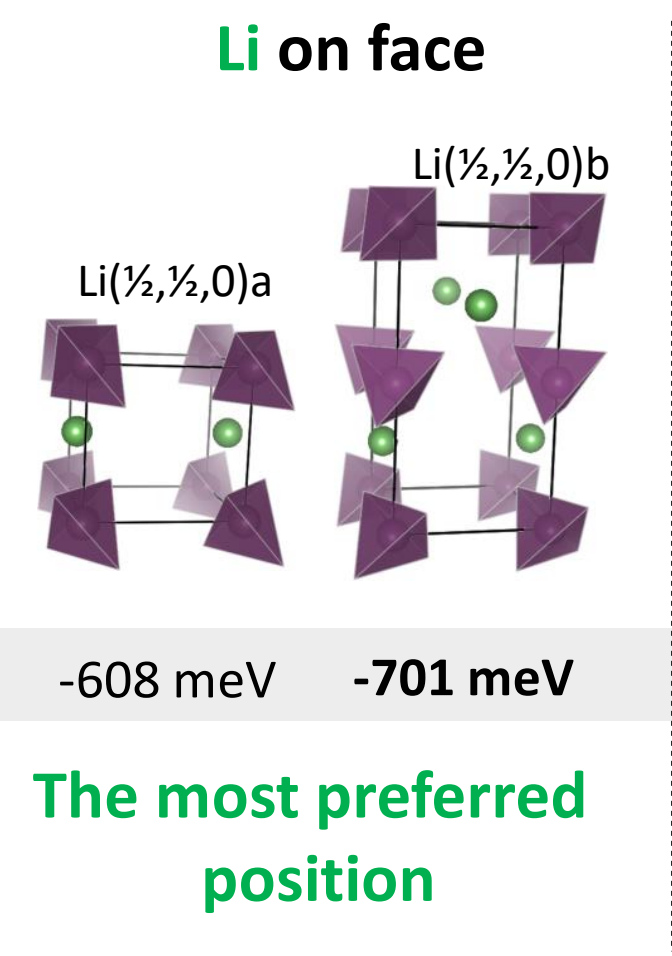

Li on edge

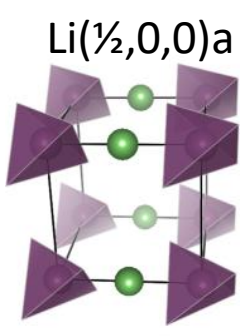

$-275 \mathrm{meV}$

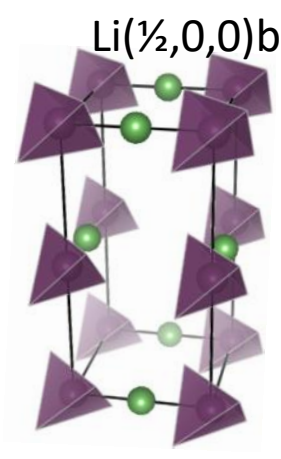

$-254 \mathrm{meV}$ 


\section{DFT Modelling $\mathrm{LiSc}\left(\mathrm{BH}_{4}\right)_{4}$ in $\mathrm{Zr}\left(\mathrm{BH}_{4}\right)_{4}$ type lattice}

Li on face

$P-42 C$

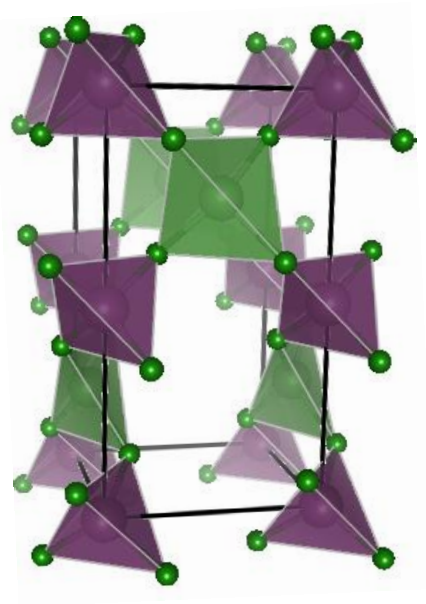

$\Delta \mathrm{E}=-701 \mathrm{meV}$

$a=5.851 \AA, c=11.827 \AA$

$d(H \ldots H)_{\text {inter }}=2.752 \AA$

DFT-D3 result
Kim et al., J. Phys. Chem. C (2009)

Kim's best model

I-4

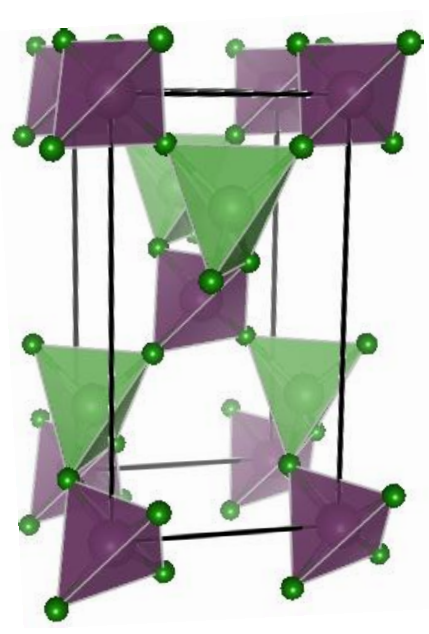

$-680 \mathrm{meV}$

$a=5.760 \AA, c=11.985 \AA$

$d(H \ldots . H)_{\text {inter }}=2.800 \AA$

DFT-D3 result
- In the lowest-E models $\mathrm{LiB}_{4}$ found in tetrahedral geometry

- The two models differ in Sc sublattice

- The model with simple cubic Sc sublattice with face-centred $\mathrm{Li}$ was found to be the ground state 


\section{DFT Modelling $\mathrm{LiSc}\left(\mathrm{BH}_{4}\right)_{4}$ in $\mathrm{Zr}\left(\mathrm{BH}_{4}\right)_{4}$ type lattice}

\section{Li on face}

$P-42 c$

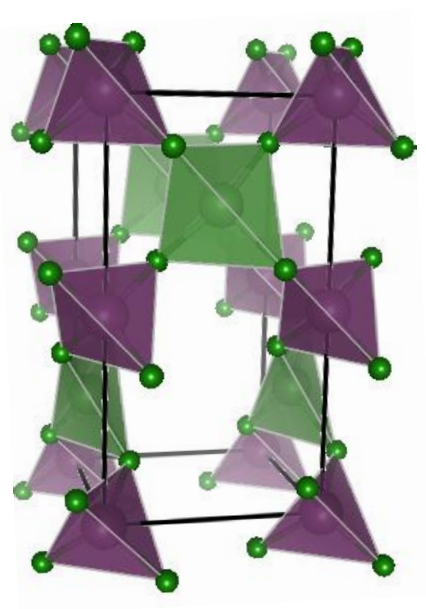

$$
\Delta \mathrm{E}=-701 \mathrm{meV}
$$

$a=5.851 \AA, c=11.827 \AA$

$d(H \ldots . . H)_{\text {inter }}=2.752 \AA$

DFT-D3 result

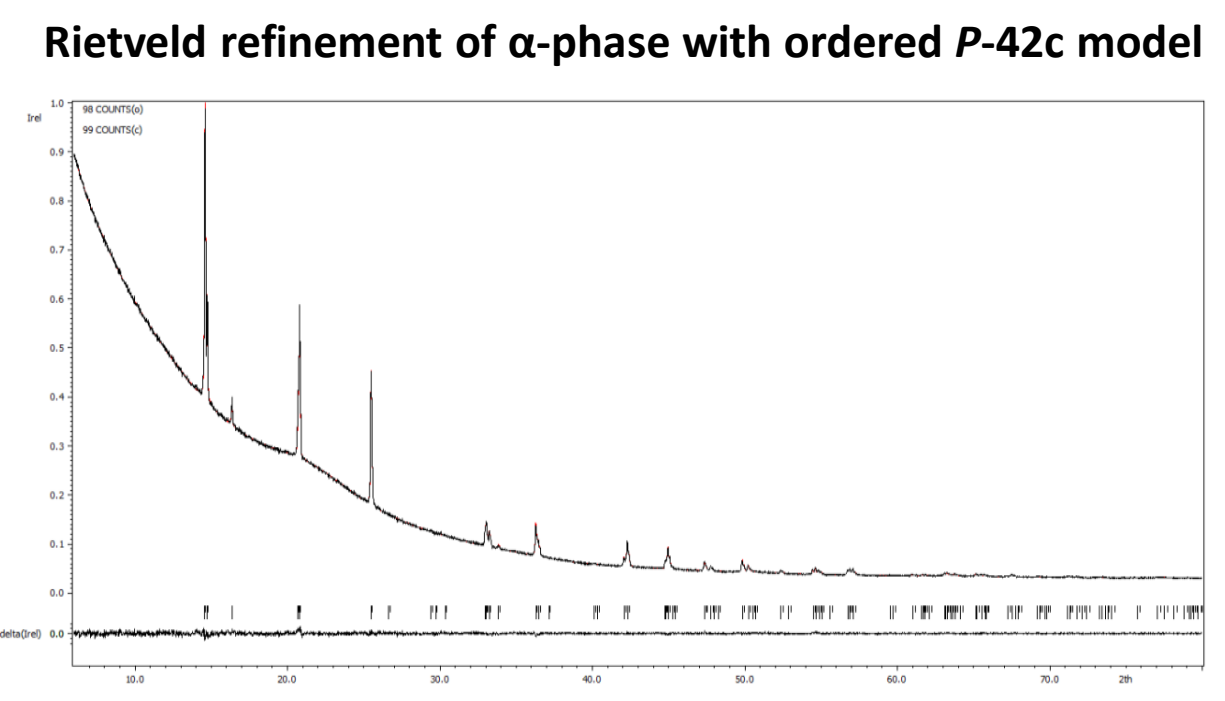

Comparison of XRD parameters:

\begin{tabular}{|c|c|c|}
\hline & $\begin{array}{c}\text { ordered } \\
\text { model }\end{array}$ & $\begin{array}{c}\text { disordered } \\
\text { model }\end{array}$ \\
\hline SPGR & P-42c & P-42c \\
\hline wRp [\%] & 1.17 & 1.44 \\
\hline cRp [\%] & 28.63 & 32.21 \\
\hline a [Å] & $6.0670(5)$ & $6.0710(8)$ \\
\hline c [Å] & $12.0147(10)$ & $12.0233(16)$ \\
\hline
\end{tabular}




\section{DFT Modelling $\mathrm{LiSc}\left(\mathrm{BH}_{4}\right)_{4}$ in $\mathrm{Zr}\left(\mathrm{BH}_{4}\right)_{4}$ type lattice}

\section{Li on face}

$P-42 C$

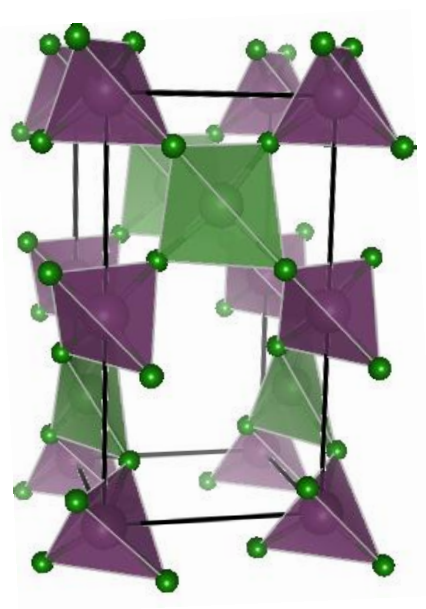

$$
\Delta \mathrm{E}=-701 \mathrm{meV}
$$

$a=5.851 \AA, c=11.827 \AA$

$d(H \ldots . . H)_{\text {inter }}=2.752 \AA$

DFT-D3 result

\section{Importance of dispersion-corrected DFT (D3)}

Simulated XRD patterns of $\alpha$

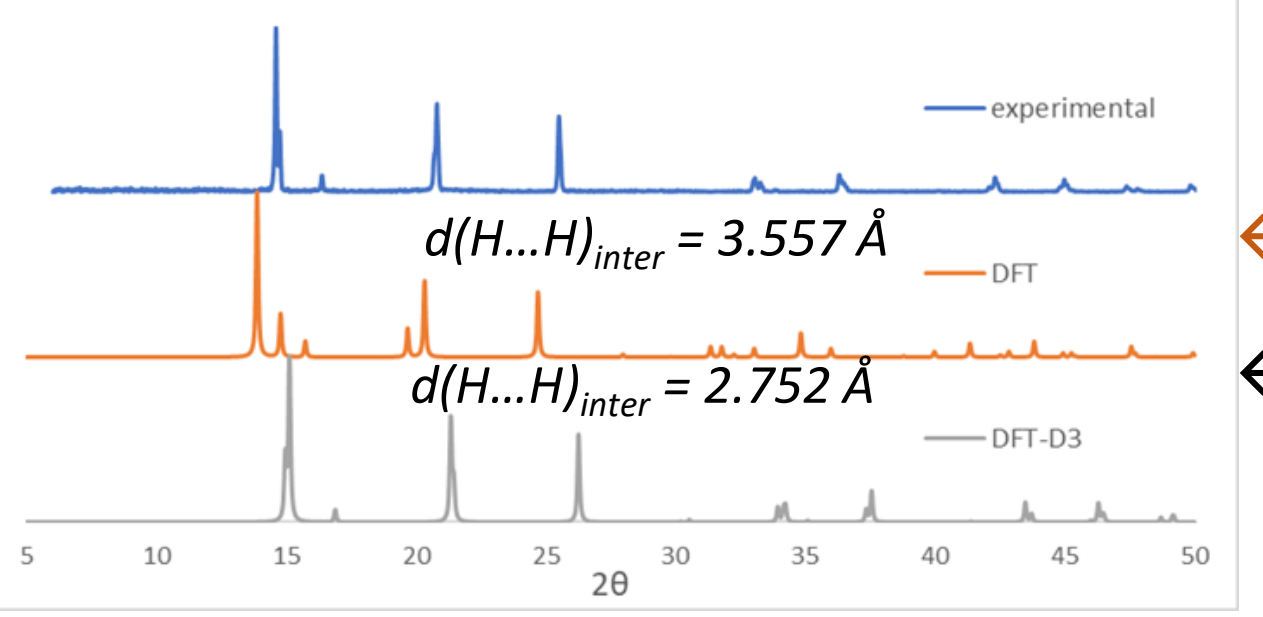

$\leftarrow$ basic DFT method fails

$\leftarrow$ Qualitative agreement reached only with dispersion-corrected DFT (DFT-D3) 


\section{Modelling $\mathrm{LiSc}\left(\mathrm{BH}_{4}\right)_{4}$ - lattice dynamics approach}

Phonon dispersion curves (DFT)

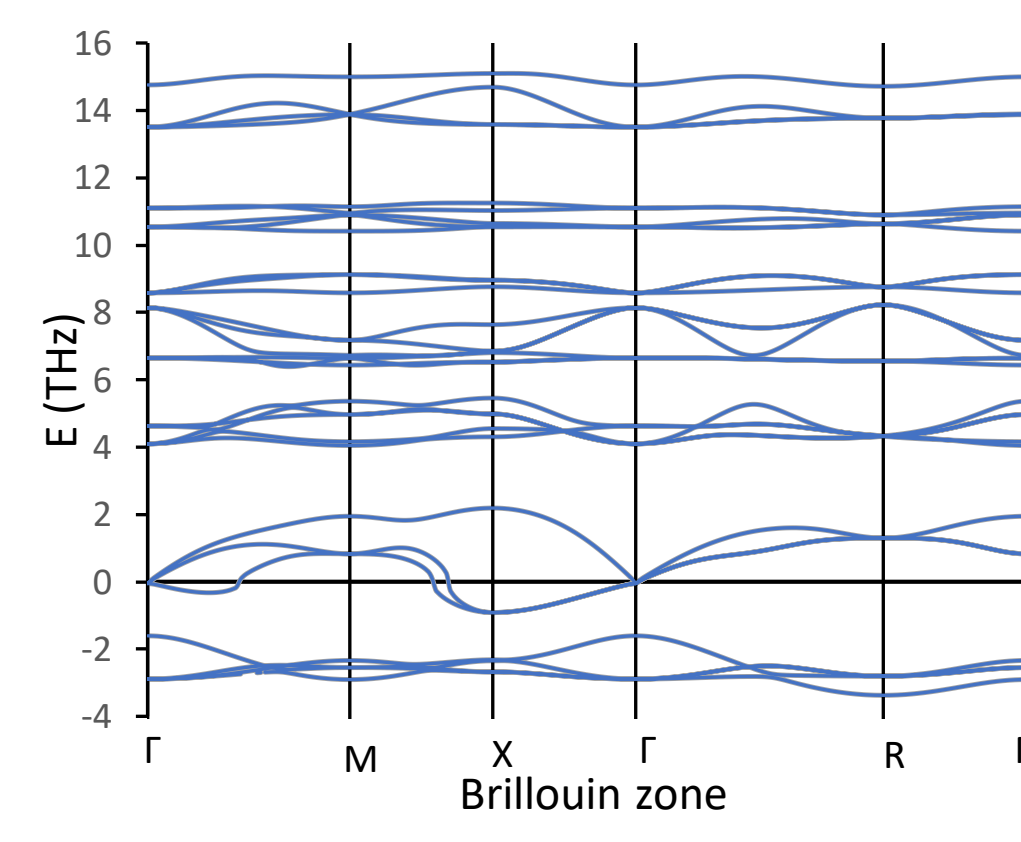

- Searched for lower-energy solutions by distorting the structure along the atomic displacements of ALL unstable

- Four optical modes are being instable:

- Li displacement,

- $\mathrm{BH}_{4}$ reorientation and translation.

- Computed close to 100 structures. unstable

modes

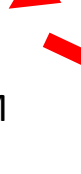

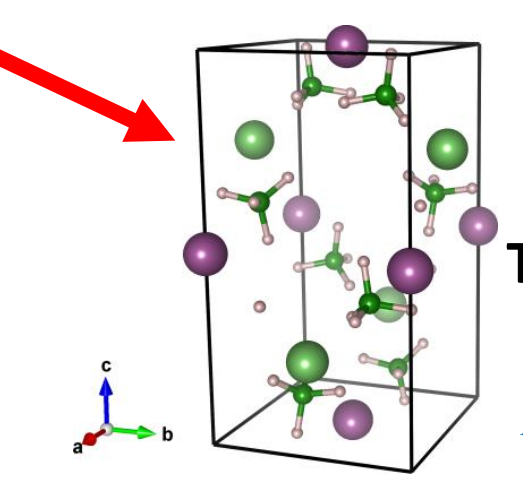
modes.

$\operatorname{Li}(1 / 2,1 / 2,1 / 2)$

$P-43 \mathrm{~m}$

The lowest-E

Kim's 'ground state' (I-4)

Kim et al., J. Phys. Chem. C (2009) 


\section{$\beta$ phase $=\operatorname{LiSc}\left(\mathrm{BH}_{4}\right)_{4-x} \mathrm{Cl}_{x}, x \approx 0.7$}
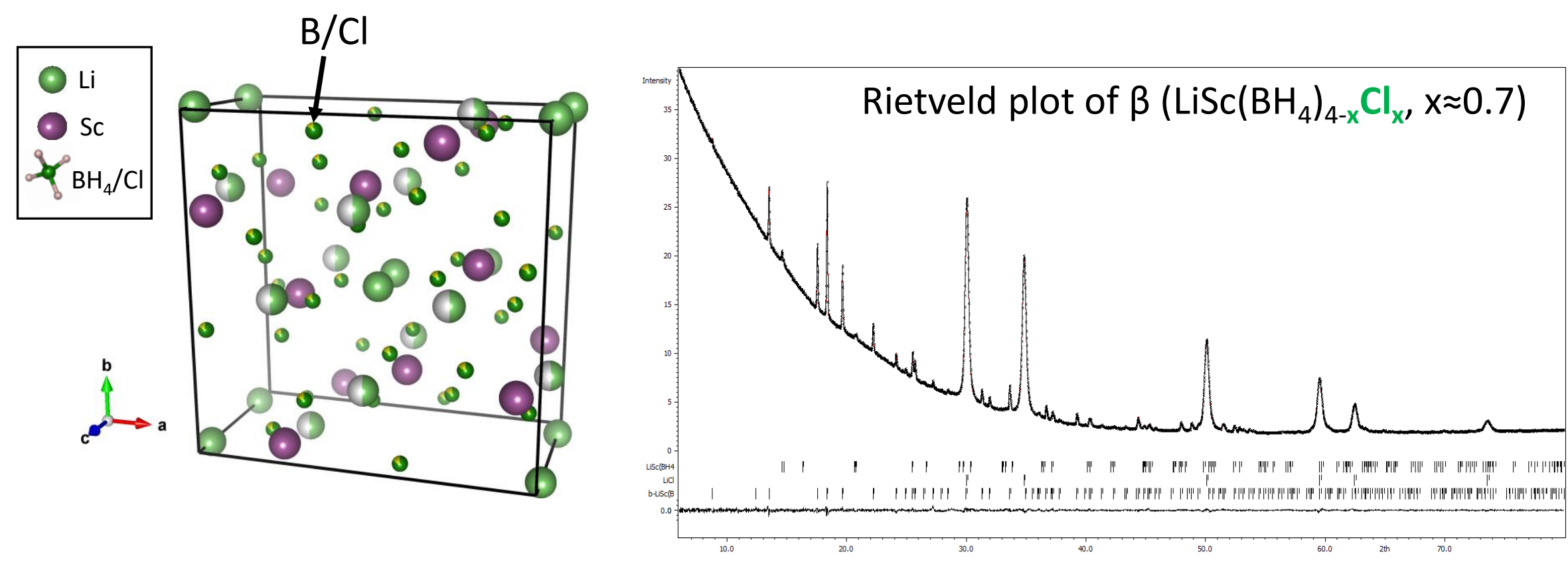

\begin{tabular}{c|c|c|}
\hline $\operatorname{LiSc}\left(\mathrm{BH}_{4}\right)_{4-x} \mathrm{Cl}_{x}$ & $x=0$ & $x \approx 0.7$ \\
\hline wRp [\%] & 0.98 & 0.97 \\
\hline cRp [\%] & 8.78 & 8.66
\end{tabular}




\section{SUMMARY}
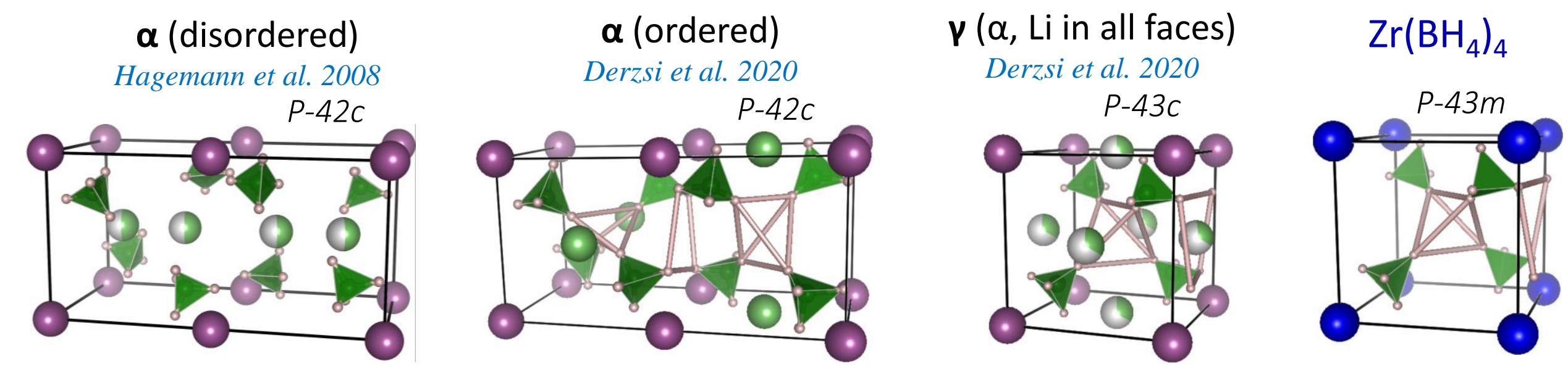

- We have been able to resolve the old standing problem of the structure and stability of the $\alpha$ polymorph.

- $\alpha$ is best described by the ordered $P-42$ c model.

- $\boldsymbol{\alpha}$ is confirmed to be the ground state (only) polymorph of $\operatorname{LiSc}\left(\mathrm{BH}_{4}\right)_{4}$.

- $\boldsymbol{\gamma}$ can be interpreted as $\boldsymbol{\alpha}$ with substantial Li disorder.

- $\beta$ redetermined as mixed anion $\mathrm{BH}_{4}-\mathrm{Cl}$ phase: $\operatorname{LiSc}\left(\mathrm{BH}_{4}\right)_{4-x} \mathrm{Cl}_{x}$, where $x \approx 0.7$. 


\section{AKCNOWLEDGEMENTS}

- Computational resources: the Interdisciplinary Centre for Mathematical and Computational Modelling, University of Warsaw under grant no. ADVANCE++ (GA76-19).

- The European Regional Development Fund, Research and Innovation Operational Programme, project No. ITMS2014+: 313011W085.

- Polish National Science Centre under grant HYDRA no. 2014/15/B/ST5/05012.

- Biopolymers Laboratory, Faculty of Physics, University of Warsaw, for the access to Agilent Supernova $\mathbf{X}$-ray single-crystal diffractometer, co-financed by the European Union within the ERDF Project POIG.02.01.00-14-122/09. 


\section{THANK YOU FOR YOUR ATTANTION}

